\title{
DIMENSIONAL REDUCTION OF NONLINEAR TIME DELAY SYSTEMS
}

\author{
M. S. FOFANA
}

Received 17 October 2002 and in revised form 15 June 2003

Whenever there is a time delay in a dynamical system, the study of stability becomes an infinite-dimensional problem. The centre manifold theorem, together with the classical Hopf bifurcation, is the most valuable approach for simplifying the infinite-dimensional problem without the assumption of small time delay. This dimensional reduction is illustrated in this paper with the delay versions of the Duffing and van der Pol equations. For both nonlinear delay equations, transcendental characteristic equations of linearized stability are examined through Hopf bifurcation. The infinite-dimensional nonlinear solutions of the delay equations are decomposed into stable and centre subspaces, whose respective dimensions are determined by the linearized stability of the transcendental equations. Linear semigroups, infinitesimal generators, and their adjoint forms with bilinear pairings are the additional candidates for the infinite-dimensional reduction.

\section{Introduction}

Time delay problems are frequently encountered in control devices (see $[4,5]$ ), machinetool chatter (see $[2,7,15,17]$ ), and building structures subject to earthquakes (see [16]). Delay differential equations (DDEs) are generally the best choice over ordinary differential equations for the study of stability of the occurring dynamics.

Suppose a dynamical system with a time delay is described by DDEs of the form

$$
\ddot{x}(t)+2 \delta \omega_{0} \dot{x}(t)+\omega_{0}^{2} x(t)+\omega_{0}^{2} \mu(x(t)+x(t-\tau))=0,
$$

where the real values $\delta, \omega_{0}$ are the damping factor and natural frequency with $\delta=c / 2 \sqrt{m k}$ and $\omega_{0}=\sqrt{k / m}$, where $m$ is the model mass, $c$ and $k$ are the viscous damping and stiffness coefficients, respectively. We define $\mu=k_{1} / k$ as a bifurcation parameter, where $k_{1}$ denotes a force coefficient, which can vary in accordance with the material of the model elements and the proportional and time delay control forces applied at the period of steady state motion. When $\mu=0$, the delay equation (1.1) becomes an ODE of the form

$$
\ddot{x}(t)+2 \delta \omega_{0} \dot{x}(t)+\omega_{0}^{2} x(t)=0 .
$$


The linearized stability study of (1.1) and (1.2) usually begins with the substitution of the trial solutions $x(t)=e^{\lambda t}, x(t-\tau)=e^{\lambda(t-\tau)}, \dot{x}(t)=\lambda e^{\lambda t}$ into the differential equations. This will yield the transcendental and algebraic characteristic equations accordingly,

$$
\begin{aligned}
& \Delta_{\mathrm{DDE}}:=\lambda^{2}+2 \delta \omega_{0} \lambda+\omega_{0}^{2}\left(1+\mu\left(1+e^{-\tau \lambda}\right)\right)=0, \\
& \Delta_{\mathrm{ODE}}:=\lambda^{2}+2 \delta \omega_{0} \lambda+\omega_{0}^{2}=0 \text {. }
\end{aligned}
$$

It can be readily seen that $\Delta_{\mathrm{DDE}}=0$ has infinite number of eigenvalues due to the transcendental exponent $e^{-\tau \lambda}$, while the algebraic equation $\Delta_{\mathrm{ODE}}=0$ has a finite number of eigenvalues. Incidentally, there will be inherent qualitative differences between the stability exchanges of the differential equations (1.1) and (1.2). Difficulty in dealing with DDEs customarily leads to the use of conventional asymptotic techniques, such as the (i) Taylor series expansion, (ii) perturbation method, and (iii) harmonic balancing under the assumption of small time delays. During their application, small errors that are generated in the inclusion of, say the first and second terms of the series expansion can be magnified upon the addition of the third- or higher-order terms. The publications by Èl'sgol'ts and Norkin [6], Kurzweil [13], and Mazanov and Tognetti [14] include some illustrations on the inconsistencies generated by using the Taylor series expansion of $x(t-\tau)=x(t)-(1 !)^{-1} \tau \dot{x}(t)+(2 !)^{-1} \tau^{2} \ddot{x}(t)-\cdots$ to convert DDEs to ODEs under the assumption of small delay $\tau>0$. The presence of time delay in a differential equation requires that an initial continuously differentiable function be defined in the domain $[-\tau, 0]$. Such an initial function forms a basis for the infinite-dimensional solutions of the DDEs in the range $\mathbb{R}^{n}$. Apparently, the stability state of a delay system at time $t$ is very much dependent on the stability state at an earlier time $t-\tau$. One must know the solution behaviour of the delay system at $x_{t}(\theta),-\tau \leq \theta \leq 0$ in order to determine the future solution behaviour $x(t+\theta), t \geq 0$. The graphs of the solutions at time $t$ and $t-\tau$ are connected by means of the fundamental definition $x_{t}(\theta)=x(t+\theta),-\tau \leq \theta \leq 0$. Uniqueness and existence of the solutions of the DDEs follow directly with the specification of the initial function. The infinite-dimensional reduction of DDEs to finite-dimensional under the assumption of small time eliminates such a requirement.

Alternatively, this paper follows the work presented in $[10,11,12]$ to reduce a class of nonlinear DDEs in the infinite-dimensional space $C\left([-\tau, 0], \mathbb{R}^{n}\right)$. It is our aim to derive equivalent centre manifold ODEs for the DDEs in $C\left([-\tau, 0], \mathbb{R}^{n}\right)$ without the assumption of small time delay $\tau>0$. The dimension of the ODEs and critical model parameters for stability exchanges as the eigenvalues of transcendental characteristic equations cross from left to right in the complex plane are determined by linearized analysis of the DDEs at a Hopf bifurcation.

\section{Dimensional reduction}

The centre manifold theorem is a means of reducing high-dimensional systems into lower-dimensional forms, while at the same time preserving the inherited dynamics of the original systems. A valuable tool for classifying dimensions of systems is Hopf bifurcation theorem $[9,12]$. The word Hopf bifurcation is in recognition of E. Hopf's contribution in 1942 to the study of periodic solutions of ODEs under some prescribed conditions. 
The conditions state that among a known $n$-number of eigenvalues associated with linearized stability analysis of a nonlinear dynamical system at a particular equilibrium point, there is a pair of complex-conjugate eigenvalues crossing the imaginary axis with a nonzero velocity, and while the remaining $n-2$ eigenvalues will lie in the left-hand side of the complex plane as a parameter crosses a critical value. There are explicit formulas for calculating the corresponding periodic solutions. The dimensional reduction of DDEs in the space $C\left([-\tau, 0], \mathbb{R}^{n}\right)$ will be carried out without violating the Hopf conditions. Notations and mathematical arguments to be presented are taken from $[3,10,11,12]$.

The specific DDEs considered are of the form

$$
\dot{x}(t)=f\left(x_{t}(\theta), \mu, \varepsilon\right), \quad x(t) \in \mathbb{R}^{n}, x_{t}(\theta) \in C:=C\left([-\tau, 0], \mathbb{R}^{n}\right), 0 \leq \varepsilon \ll 1,
$$

where for any given initial continuously differential function $\phi(\theta) \in C$ equipped with the usual supremum norm $\|\cdot\|$ in $C$ and vector norm $|\cdot|$ in $\mathbb{R}^{n}$ defined by $\|\phi(\theta)\|=$ $\sup _{-\tau \leq \theta \leq 0}|\phi(\theta)|$, one can state the condition $\phi(\theta)=x(\theta),-\tau \leq \theta \leq 0 . f=f\left(x_{t}(\theta), \mu, \varepsilon\right)$ : $\mathbb{R} \times C \rightarrow \mathbb{R}^{n}$ is nonlinear and continuous with respect to its arguments. The element $x_{t}(\theta) \in C$ is the past history state for all time $t$ of the future state $x(t) \in \mathbb{R}^{n}$ up to time $t=0$ for which $x_{t}(\theta)$ is defined in the domain $[-\tau, 0]$, and then translated forward onto the range $\mathbb{R}^{n}$ through the fundamental definition $x_{t}(\theta)=x(t+\theta),-\tau \leq \theta \leq 0$. Essentially, a function $x=x(t, \mu, \varepsilon) \in \mathbb{R}^{n}$ is a solution of (2.1) through $\phi(\theta)$ with initial value $\phi(0)$ at zero if and only if for $t \in[-\tau, \infty)$ the function $x_{t}=x_{t}(\theta, \mu, \varepsilon) \in C$ exists and is a solution of (2.1). We will find the solution of (2.1) in $C$ for $\tau>0$ as the parameter $\mu$ is varied near some critical value $\mu_{c}$.

We assume that there is an equilibrium point, which for simplicity is the trivial solution $x_{t}(\theta)=0$ of (2.1). At this equilibrium point, we further assume that $f(0, \mu, 0)=$ $\partial f(0, \mu, 0) / \partial \phi(\theta)=0$ and the Fréchet derivative $\partial f(0, \mu, 0) / \partial \phi(\theta)$ of $f$ with respect to $\phi(\theta) \in C$ is given by $\partial f(0, \mu, 0) / \partial \phi(\theta) \equiv L(\phi(\theta), \mu) \phi(\theta)$, where $L=L\left(x_{t}(\theta), \mu\right): C \times \mathbb{R} \rightarrow$ $\mathbb{R}^{n}$ is a linear functional mapping. With the representation $\Delta f=\Delta f(\phi(\theta), \mu, \varepsilon): \mathbb{R} \in$ $C \rightarrow \mathbb{R}^{n}$, we define $f$ in $(2.1)$ as $f=L(\phi(\theta), \mu) \phi(\theta)+\varepsilon \Delta f(\phi(\theta), \mu, \varepsilon)$, where $\Delta f$ is strictly a nonlinear functional mapping. Furthermore, it is known by the Riesz representation theorem that the linear functional mapping $L$ can be written in terms of a function of bounded variation, denoted by $\eta(\theta, \mu):[-\tau, 0] \rightarrow \mathbb{R}^{n}$, and a Riemann-Stieltjes integral type. To this end, we have the variational DDEs of the form

$$
\dot{x}(t)=L(\phi(\theta), \mu) \phi(\theta)+\varepsilon \Delta f(\phi(\theta), \mu, \varepsilon), \quad \Delta f(\phi(\theta), \mu, \varepsilon): \mathbb{R} \times C \longrightarrow \mathbb{R}^{n},
$$

where the Fréchet derivative $\partial f(0, \mu, 0) / \partial \phi(\theta)$ of $f$ is given by

$$
\begin{gathered}
\frac{\partial f(0, \mu, 0)}{\partial \phi(\theta)}=L(\phi(\theta), \mu) \phi(\theta):=\int_{-\tau}^{0}[d \eta(\theta, \mu)] \phi(\theta), \quad \phi(\theta) \in C, \\
\eta(\theta, \mu)= \begin{cases}L(\phi(-\tau), \mu), & \theta=-\tau, \\
0, & -\tau<\theta<0, \\
L(\phi(0), \mu), & \theta=0 .\end{cases}
\end{gathered}
$$


The function $x(t, \mu, \varepsilon) \in \mathbb{R}^{n}$ is the nonlinear solution of (2.2), provided that $x_{t}(\theta, \mu, \varepsilon) \in C$ satisfies the variation of constant-integral equation

$$
x_{t}(\theta, \mu, \varepsilon)=J(t, \mu) \phi(\theta)+\varepsilon \int_{0}^{t} J((t-\xi), \mu) \times X_{0}(\theta) \triangle f\left(x_{t}(\theta), \mu, \varepsilon\right) d \xi, \quad t \in(-\infty, \infty),
$$

in $C$, where the element $X_{0}(\theta)$ is $n \times n$ matrix function, defined as

$$
X(\theta)= \begin{cases}0 & -\tau \leq \theta<0 \\ I & \theta=0\end{cases}
$$

and $I$ is an identity operator. In the integral equations $(2.3), J(t, \mu), t, \mu \geq 0$ is a semigroup of bounded linear operators with infinitesimal generator $A(\theta, \mu) \in C$ defined with the aid of the linearized delay equation

$$
\dot{x}(t)=\int_{-\tau}^{0}[d \eta(\theta, \mu)] \phi(\theta), \quad x(\theta)=\phi(\theta),-\tau \leq \theta \leq 0,
$$

as follows:

$$
\begin{aligned}
& D(A(\theta, \mu))=\left\{\phi(\theta) \in C: \frac{d \phi(\theta)}{d \theta} \in C,\left.\frac{d \phi(\theta)}{d \theta}\right|_{\theta=0}=\int_{-\tau}^{0}[d \eta(\theta, \mu)] \phi(\theta)\right\}, \\
& A(\theta, \mu) \phi(\theta)=\frac{d \phi(\theta)}{d \theta}
\end{aligned}
$$

where $\phi(\theta)$ is in the domain $D(A(\theta, \mu))$. The semigroup $J(t, \mu)$ maps $C$ into itself, namely, $J(t, \mu): C \in \mathbb{R} \rightarrow C$, that is, a linear solution of $(2.2)$, defined in the domain $[-\tau, 0]$ can be carried over to the range $\mathbb{R}^{n}$ by the relation $x_{t}(\phi(\theta), \mu)=J(t, \mu) \phi(\theta)$, for $t, \mu \geq 0$. Generally, the explicit nature of $J(t, \mu)$ is not known, but in view of its linearity, compactness, and the fact that $J(0, \mu)=I$ and $J(t, \mu) J(\varsigma, \mu)=J(t+\varsigma, \mu)$ for $t, \varsigma \geq 0$, one can infer that the point spectral sets $\sigma(J(t, \mu))$ of $J(t, \mu)$ and $\sigma(A(\theta, \mu))$ of the generator $A(\theta, \mu) \in C$ are exactly the eigenvalues $\lambda$ of the transcendental characteristic equation

$$
\Delta(\lambda, \mu):=\operatorname{det}\left\{\lambda I-\int_{-\tau}^{0}[d \eta(\theta, \mu)] e^{\lambda \theta}\right\}=0
$$

which may be real or occur in complex conjugates as the parameter $\mu$ varies.

We will assume that at the critical value $\mu_{c}$ of the bifurcation parameter $\mu$, the characteristic equation $\Delta(\lambda, \mu)=0$ has the eigenvalues $\lambda_{1,2}=v(\mu) \pm i \omega(\mu), v(\mu)>0, \omega(\mu) \neq 0$, satisfying Hopf bifurcation conditions $v\left(\mu_{c}\right)=0, \omega\left(\mu_{c}\right) \neq 0, \mathbb{R} e\{d \Delta(\lambda, \mu) / d \mu\} \neq 0$. No other eigenvalues, in particular, for any integer $\kappa, \pm i \kappa \omega(\mu)$, are on the imaginary axis. All the remaining eigenvalues of $\Delta(\lambda, \mu)=0$ have negative real parts. Then, there exists the direct sum decomposition of $C=P \oplus Q$ by all eigenvalues of $\Delta(\lambda, \mu)=0$, where the subspace $P=P(\lambda, \mu) \in C$ is the centre generalized eigenspace corresponding to the eigenvalues $\lambda_{1,2}= \pm i \omega(\mu), i=\sqrt{-1}$ and $Q=Q(\lambda, \mu) \in C$ is the infinite-dimensional complementary subspace associated with the remaining eigenvalues of $\Delta(\lambda, \mu)=0$. Furthermore, tangent to the generalized subspace $P$ is a parabolic smooth curve representing 
a local centre manifold $M_{\mu}^{P}=M_{\mu}^{P}(A(\theta, \mu)) \in C\left([-\tau, 0], \mathbb{R}^{n}\right)$ in which interesting dynamics of (2.2) can be explored via studying the solutions of the centre manifold ODEs. The subspaces $P$ and $Q$ are disjoint and invariant under $J(t, \mu)$ and its generator $A(\theta, \mu)$. The invariant means that a solution in $C$ starting from a point in either $P$, or $Q$, will indeed always remain in $P$ for all $t \in(-\infty, \infty)$, or in $Q$ for all $t \in[0, \infty)$. Also, it is interesting to note that $A(\theta, \mu) M_{\mu}^{P}(A(\theta, \mu)) \subset M_{\mu}^{P}(A(\theta, \mu))$, and there exists a $\kappa \times \kappa$ constant matrix $B \in C$ whose elements are the eigenvalues of $\Delta(\lambda, \mu)=0$ with zero real parts. The values of $\kappa=1,2, \ldots, n$ correspond to the multiplicity of the eigenvalues of the point spectra of the semigroup $J(t, \mu)$ and its generator $A(\theta, \mu)$.

For the particular eigenvalues $\lambda_{1,2}= \pm i \omega(\mu)$ of (2.5), the centre manifold $M_{\mu}^{P}$ is of dimension 2 . We denote the linearly independent solutions for $\lambda_{1,2}= \pm i \omega(\mu)$ by the matrix function $\Phi(\theta)=\left[\phi_{1}(\theta), \phi_{2}(\theta)\right] \in C$, whose elements are symbolically denoted by $\phi_{1}(\theta)=\left[\phi_{11}(\theta), \phi_{21}(\theta)\right]^{T}, \phi_{2}(\theta)=\left[\phi_{12}(\theta), \phi_{22}(\theta)\right]^{T}$, where $T$ stands for transpose. This matrix function $\Phi(\theta) \in C$ forms a basis for $P \in C$ or for all the solutions to (2.2) on the centre manifold $M_{\mu}^{P}$. Moreover, $\Phi(\theta)$ constitutes the elements of the initial continuous function, namely, $\phi(\theta)=\Phi(\theta) b,-\tau \leq \theta \leq 0$, where $b$ is some constant vector.

To determine the basis for the stable complementary subspace $Q \in C$, we further consider the delay equation

$$
\dot{u}(s)=\hat{L}(\psi(s), \mu) \psi(s)=-\int_{-\tau}^{0}[d \hat{\eta}(s, \mu)] \psi(-s), \quad u_{\hat{t}}(s)=\psi(s), \quad 0 \leq s \leq \tau,
$$

in the space $\hat{C}:=\hat{C}\left([0, \tau], \mathbb{R}^{n}\right)$ and along with its semigroup $J(\hat{t}, \mu), \hat{t}, \mu \geq 0$ and infinitesimal generator $\widehat{A}(s, \mu)$ defined for $\psi(s) \in D(\widehat{A}(s, \mu))$ as

$$
\begin{aligned}
& D(\hat{A}(s, \mu))=\left\{\psi(s) \in \hat{C}: \frac{d \psi(s)}{d s} \in \hat{C},\left(\frac{d \psi(s)}{d s}\right)_{s=0}=-\int_{-\tau}^{0}[d \hat{\eta}(s, \mu)] \psi(-s)\right\}, \\
& \widehat{A}(s, \mu) \psi(s)=-\frac{d \psi(s)}{d s},
\end{aligned}
$$

which are formally adjoint to (2.4) with respect to the bilinear relation

$$
\left(\psi_{j}(s), \phi_{\kappa}(\theta)\right)=\left(\psi_{j}(0), \phi_{k}(0)\right)-\int_{-\tau}^{0} \int_{0}^{\theta} \psi_{j}(\xi-s)[d \eta(\theta, \mu)] \phi_{\kappa}(\xi) d \xi, \quad j, \kappa=1,2,3, \ldots, n,
$$

in $C \times \hat{C}$, where $\phi_{\kappa}(\theta) \in C, \psi_{j}(s) \in \hat{C}$ and $\hat{\eta}(s, \mu):[0, \tau] \rightarrow \mathbb{R}^{n}$ is the transpose of $\eta(\theta, \mu)$ : $[-\tau, 0] \rightarrow \mathbb{R}^{n}$. The properties of the semigroup $J(\hat{t}, \mu)$ and its generator $\hat{A}(s, \mu)$ of the adjoint equations (2.6) are the same as those of $J(t, \mu)$ and $A(\theta, \mu)$ in $C$. Essentially, the point spectral sets of $J(\hat{t}, \mu)$ and $\hat{A}(s, \mu)$ are of the finite type and they have eigenvalues satisfying the adjoint transcendental characteristic equation $\widehat{\Delta}(\lambda, \mu):=\operatorname{det}\{\lambda I-$ $\left.\int_{-\tau}^{0}[d \hat{\eta}(s, \mu)] e^{-\lambda s}\right\}=0$. These eigenvalues $\lambda$ of $\widehat{\Delta}(\lambda, \mu)=0$ are exactly those of $\Delta(\lambda, \mu)=0$ in $C$. Then, in the same way as before, we have the centre generalized eigenspace $\hat{P}=$ $\widehat{P}(\lambda, \mu) \in \widehat{C}$ and the centre manifold $\widehat{M}_{\mu}^{\hat{P}}=\widehat{M}_{\mu}^{\hat{P}}(\widehat{A}(s, \mu)) \in \widehat{C}$ for the eigenvalues $\lambda_{1,2}=$ $\pm i \omega(\mu)$ at $\mu=\mu_{c} . \widehat{P}$ and $\widehat{M}_{\mu}^{\hat{P}}$ are both invariant under $J(\hat{t}, \mu)$ and $\widehat{A}(s, \mu)$, and there exists 
also a constant matrix $\widehat{B}$, whose elements are the real values of $\widehat{\Delta}(\lambda, \mu)=0$. The corresponding basis for $\widehat{P}$ is the matrix function $\Psi(s)=\left[\psi_{1}(s), \psi_{2}(s)\right]^{T} \in \hat{C}$ whose elements are denoted symbolically by the form $\psi_{1}(s)=\left[\psi_{11}(s), \psi_{21}(s)\right]^{T}, \psi_{2}(s)=\left[\psi_{12}(s), \psi_{22}(s)\right]^{T}$. Again, with some constant vector $\hat{b}$, we have the required initial function $\psi(s)=\Psi(s) \hat{b}$ in $\widehat{C}$ for all the solutions to the adjoint delay equations (2.6).

Having the bases $\Phi(\theta) \in C, \Psi(s) \in \hat{C}$ for $P \in C, \hat{P} \in \hat{C}$, and the generators $A(\theta, \mu) \in C$, $\widehat{A}(s, \mu) \in \hat{C}$ as above, the constant matrices $B \in C$ and $\widehat{B} \in \hat{C}$ can be derived as follows. It is known that $A(\theta, \mu) \Phi(\theta)=\Phi(\theta) B,-\tau \leq \theta \leq 0$, and $\hat{A}(s, \mu) \Psi(s)=\Psi(s) \hat{B}, 0 \leq s \leq \tau$. The relation $A(\theta, \mu) \Phi(\theta)=\Phi(\theta) B$ at Hopf bifurcation yields $\Phi(\theta)=\Phi(0) e^{B \theta},-\tau \leq \theta \leq 0$ with $B=\left[[0, \omega]^{T},[-\omega, 0]^{T}\right]$. Incidentally, $J(t, \mu) \phi(\theta)=\Phi(0) e^{B(t+\theta)} b, t \geq 0$ is the solution operator of the linearized delay equations (2.4). By the definition of the adjoint solutions of the DDEs in $C \times \hat{C}$, it is further known that $(\Psi(s), A(\theta, \mu) \Phi(\theta))=(\widehat{A}(s, \mu) \Psi(s), \Phi(\theta))$, where using the identities $(\Psi(s), A(\theta, \mu) \Phi(\theta))=(\Psi(s), \Phi(\theta)) B$ and $(\hat{A}(s, \mu) \Psi(s), \Phi(\theta))=$ $\hat{B}(\Psi(s), \Phi(\theta))$, one can see that $(\Psi(s), \Phi(\theta)) B=\hat{B}(\Psi(s), \Phi(\theta))$. From this, it readily follows that $B=\hat{B}$, if and only if the inner product matrix $(\Psi(s), \Phi(\theta))=I$, is the identity matrix. The elements of the inner product matrix $(\Psi(s), \Phi(\theta))$ are the bilinear pairing in (2.6c), namely, $(\Psi(s), \Phi(\theta))=\left(\psi_{j}(s), \phi_{\kappa}(\theta)\right), j, \kappa=1,2$. Oftentimes, the value of $(\Psi(s), \Phi(\theta))$ is different from being the identity matrix, that is, $(\Psi(s), \Phi(\theta)) \neq I$. In such a situation, one can normalize the adjoint basis $\Psi(s)$ for $\hat{P} \in \hat{C}$ to a new basis function $\bar{\Psi}(s) \in \hat{C}$ by computing $\bar{\Psi}(s)=(\bar{\Psi}(s), \Phi(\theta))^{-1} \Psi(s)$. Then, the substitution of the new bilinear elements $\left(\bar{\psi}_{j}(s), \phi_{\kappa}(\theta)\right), j, \kappa=1,2$ of $(\bar{\Psi}(s), \Phi(\theta))$ into (2.6c) will yield $(\bar{\Psi}(s), \Phi(\theta))$ $=I$, and thus $B=\hat{B}$.

Since the elements $\phi(\theta), X_{0}(\theta), x_{t}(\phi(\theta), \mu, \varepsilon)$ in the variation-of-constant integral equations (2.3) are contained in $C$, the decomposition of $C$ as $C=P \oplus Q$ yields the unique representations

$$
x_{t}(\theta, \mu, \varepsilon)=x_{t}^{P}(\phi(\theta), \mu, \varepsilon)+x_{t}^{Q}(\phi(\theta), \mu, \varepsilon),
$$

where

$$
\begin{array}{ll}
x_{t}^{P}(\theta, \mu, \varepsilon)=J(t, \mu) \phi^{P}(\theta)+\varepsilon \int_{0}^{t} J((t-\xi), \mu) \times X_{0}^{P}(\theta) \triangle f\left(x_{t}(\theta), \mu, \varepsilon\right) d \xi, & t \in(-\infty, \infty), \\
x_{t}^{Q}(\theta, \mu, \varepsilon)=J(t, \mu) \phi^{Q}(\theta)+\varepsilon \int_{0}^{t} J((t-\xi), \mu) \times X_{0}^{Q}(\theta) \triangle f\left(x_{t}(\theta), \mu, \varepsilon\right) d \xi, & t \in[0, \infty),
\end{array}
$$

and the elements are

$$
\begin{gathered}
\phi(\theta)=\phi^{P}(\theta)+\phi^{Q}(\theta), \quad \phi^{P}(\theta):=\Phi(\theta) b, \phi^{Q}(\theta):=\phi(\theta)-\Phi(\theta) b, \\
X_{0}(\theta)=X_{0}^{P}(\theta)+X_{0}^{Q}(\theta), \quad X_{0}^{P}(\theta)=\bar{\Psi}(0) \Phi(\theta), X_{0}^{Q}(\theta)=X_{0}(\theta)-\bar{\Psi}(0) \Phi(\theta), \\
\Phi(\theta)=\Phi(0) e^{B \theta}, \quad-\tau \leq \theta \leq 0, \quad \Psi(s)=\Psi(0) e^{-\widehat{B s}}, \quad 0 \leq s \leq \tau, \\
b=(\bar{\Psi}(s), \phi(\theta)), \quad B=\left[\begin{array}{cc}
0 & -\omega \\
\omega & 0
\end{array}\right], \quad \bar{\Psi}(0)=\left\{(\bar{\Psi}(s), \Phi(\theta))^{-1} \Psi(s)\right\}_{s=0} .
\end{gathered}
$$


At this stage, the exponential estimates of the integral solutions (2.7) can be determined. The next theorem is a candidate for the solution estimates in $C$.

Theorem 2.1 (Hale [10], Hale and Verduyn Lunel [11]). For any real number, say $\beta$, let $\wedge(\lambda, \mu)=\{\lambda \in A(\theta, \mu) \mid \Delta(\lambda, \mu)=0, \mathbb{R} e \lambda \geq \beta\}$ be the point spectral set of the finite type and have eigenvalues satisfying the characteristic equation (2.5). If $C$ is decomposed into the generalized eigenspace $P$ and complementary subspace $Q$ by all the eigenvalues of $\Delta(\lambda, \mu)=0$ as $C=P \oplus Q$, then, for any $\phi(\theta) \in C$ with the representation $\phi(\theta)=\phi^{P}(\theta)+\phi^{Q}(\theta)$, there exist positive constants $\rho$ and $\nu=\nu(\rho)$ such that the following inequalities hold:

$$
\begin{aligned}
& \left\|J(t, \mu) \phi^{P}(\theta)\right\| \leq v e^{(\beta+\rho) t}\left\|\phi^{P}(\theta)\right\|, \quad t \leq 0, \\
& \left\|J(t, \mu) \phi^{Q}(\theta)\right\| \leq v e^{-(\beta+\rho) t}\left\|\phi^{Q}(\theta)\right\|, \quad t \geq 0 .
\end{aligned}
$$

Remark 2.2. The proof of the estimation theorem is found in $[10,11]$. The inequalities (2.8) form the framework for the determination of bounds for the projected nonlinear solutions of (2.2) in $P$ and $Q$, respectively. With the solution operator $J(t, \mu) \Phi(\theta)=$ $\Phi(0) e^{B(\theta+t)}, t \geq 0$, the inequalities (2.8) will yield the estimates of the linearized solutions in $P, Q \in C$ as follows: $\left\|x_{t}^{P}(\phi(\theta), \mu)\right\| \rightarrow 0$ as $t \rightarrow-\infty,\left\|x_{t}^{P}(\phi(\theta), \mu)\right\| \neq 0$ as $t \rightarrow \infty$, and while $\left\|x_{t}^{Q}(\phi(\theta), \mu)\right\| \rightarrow 0$ when $t \rightarrow \infty$. In the same way, the inequality (2.8b) yields the estimate for the nonlinear integral solution $x_{t}^{Q}(\theta, \mu, \varepsilon)$ of $Q \in C$ as

$$
\left\|J(t, \mu) \phi^{Q}(\theta)\right\|=\left\|x_{t}^{Q}(\phi(\theta), \mu)-\varepsilon \times \int_{0}^{t} J((t-\xi), \mu) X_{0}^{Q}(\theta) \Delta f(\phi(\theta), \mu, \varepsilon) d \xi\right\|, \quad t \in[0, \infty),
$$

where the use of the elements in $(2.7 \mathrm{c})$ leads to

$$
\begin{aligned}
\left\|J(t, \mu) \phi^{Q}(\theta)\right\| & =\left\|J(t, \mu)\left\{\phi(\theta)-\phi^{P}(\theta)\right\}\right\| \\
& =\left\|J(t, \mu) \Phi(0) e^{B t} b-\Phi(0) e^{B t} b\right\| \longrightarrow 0 \\
& =\left\|x_{t}^{Q}(\phi(\theta), \mu)-\varepsilon \int_{0}^{t} J((t-\xi), \mu) X_{0}^{Q}(\theta) \Delta f(\phi(\theta), \mu, \varepsilon) d \xi\right\|,
\end{aligned}
$$

as $t \rightarrow \infty$ and $J(0, \mu)=I$. On the other hand, the inequality (2.8a) will show that the exponential estimate for $x_{t}^{P}(\phi(\theta), \mu, \varepsilon), t \in(-\infty, \infty)$ of $P$ is bounded as $t \rightarrow-\infty$ and unbounded when $t \rightarrow \infty$. The latter is indeed the solution that is equivalent to the centre manifold ODEs. Chow and Mallet-Paret [3] pointed out that solutions of DDEs of the form $x_{t}^{Q}(\phi(\theta), \mu, \varepsilon)$ in $Q$, in general, do not satisfy the fundamental property, namely, $x_{t}^{Q}(\theta) \neq x_{t}^{Q}(t+\theta),-\tau \leq \theta \leq 0$. There is no loss of generality to describe the long-term behaviour of the original nonlinear DDEs (2.2) with the corresponding set of centre manifold ODEs for $x_{t}^{P}(\phi(\theta), \mu, \varepsilon),-\tau \leq \theta \leq 0, t \in[0, \infty)$.

Consequently, the direct differentiation of $x_{t}^{P}(\phi(\theta), \mu, \varepsilon)=\Phi(\theta) y(t)+x_{t}^{Q}(\phi(\theta), \mu, \varepsilon)$, where $y(t) \in \mathbb{R}^{2}, y(t)=\left(\bar{\Psi}(s), \phi^{P}(\theta)\right)$, gives

$$
\Phi(\theta) \frac{d y(t)}{d t}=\frac{d}{d t}\left(J(t, \mu) \phi^{P}(\theta)+\varepsilon \int_{0}^{t} J((t-\xi), \mu) X_{0}^{P}(\theta) \triangle f\left(x_{t}(\theta), \mu, \varepsilon\right) d \xi\right),
$$


and using the elements in $(2.7 \mathrm{c})$ leads to

$$
\Phi(0) e^{B(\theta+t)} \frac{d y(t)}{d t}=\frac{d}{d t}\left(\Phi(0) e^{B(\theta+t)} b+\varepsilon \int_{0}^{t} \Phi(0) e^{B(\theta+t-\xi)} \bar{\Psi}(0) \triangle f(\Phi(\theta) y(t), \mu, \varepsilon) d \xi\right)
$$

which will subsequently produce the centre manifold ODEs

$$
\left[\begin{array}{l}
\dot{y}_{1} \\
\dot{y}_{2}
\end{array}\right]=\left[\begin{array}{cc}
0 & -\omega \\
\omega & 0
\end{array}\right]\left[\begin{array}{l}
y_{1} \\
y_{2}
\end{array}\right]+\varepsilon\left[\begin{array}{ll}
\bar{\psi}_{11}(0) & \bar{\psi}_{12}(0) \\
\bar{\psi}_{12}(0) & \bar{\psi}_{22}(0)
\end{array}\right]\left[\begin{array}{c}
0 \\
\Delta f\left(\Phi(\theta) y(t)+x_{t}^{Q}(\theta, \mu, \varepsilon), \mu, \varepsilon\right)
\end{array}\right],
$$

for all values of $t \in[-\tau, \infty)$. The variation-of-constant integral equation $x_{t}^{Q}(\theta, \mu, \varepsilon)$ in the infinite-dimensional complementary space $Q \in C$ is equivalent to the operator differential equation

$$
\begin{aligned}
\frac{d x_{t}^{Q}(\theta, \mu, \varepsilon)}{d t} & =A_{Q}(\theta, \mu)\left(x_{t}^{Q}(\theta)+\varepsilon X_{0}^{Q}(\theta)\right)(\theta) \triangle f\left(x_{t}^{Q}(\theta), \mu, \varepsilon\right) \\
& =A_{Q}(\theta, \mu)\left(x_{t}(\theta)-x_{t}^{P}(\theta)\right)+\varepsilon\left(X_{0}(\theta)-\bar{\Psi}(0) \Phi(\theta)\right) \triangle f\left(x_{t}(\theta), \mu, \varepsilon\right),
\end{aligned}
$$

where the infinitesimal generator $A_{Q}$ has eigenvalues restricted to $Q$ and none of these eigenvalues are of the form $\pm i \kappa \omega(\mu)$ for all integers $\kappa$. In the ODEs (2.11), the time delay $\tau$ now appears as a coefficient, and the study of stability can be carried out without much difficulty. The next sections contain specific applications of the aforementioned dimensional reduction approach.

\section{Duffing equation with delay}

Consider the delay differential equation

$$
\ddot{x}+2 \delta \omega_{0} \dot{x}+\omega_{0}^{2} x+\omega_{0}^{2} \mu(x+x(t-\tau))+\varepsilon \omega_{0}^{2} \sigma_{3}(x+x(t-\tau))^{3}=0,
$$

for which $0 \leq \varepsilon \ll 1$, we define $\varepsilon \sigma_{3}=\gamma_{3} / k$ and $\gamma_{3}$ is a force coefficient due to the cubic nonlinearity. Whenever a damped or undamped motion of a dynamical system with nonlinear spring is of interest, the Duffing equation has always come handy. The equation has led to important nonlinear phenomena, connecting mathematical theories of nonlinear dynamics and applications. It is an important equation, which most undergraduate and graduate students learn to solve fundamental problems of nonlinear dynamics. As seen in (3.1), the equation is modified by adding the time delay $\tau$ as a means to trigger instability in an otherwise stable system when the model parameters vary and pass critical values. This modification presents an infinite-dimensional problem, and from the perspectives on modelling, phenomena, and analysis, it provides an opportunity for more dimensional variability in the study of stability. It is indeed amenable to new phenomenological dynamics and computational tools at the interface of delay differential equations and the theories of nonlinear dynamics in higher-dimensional settings. The goal here is to determine critical values for $\mu$ and $\tau$ when stability exchanges take place at 
some desired frequency and damping factor $0<\delta<1$. We begin by first focusing on the linearized analysis of the delay equation (3.1) at a Hopf bifurcation point.

3.1. Linear analysis. The linearized delay equation when $\varepsilon=0$,

$$
\ddot{x}+2 \delta \omega_{0} \dot{x}+\omega_{0}^{2}(1+\mu) x+\omega_{0}^{2} \mu x(t-\tau)=0, \quad \mu=\mu_{c}+\varepsilon \tilde{\mu},
$$

in $C:=C\left([-\tau, 0], \mathbb{R}^{n}\right)$ and its adjoint form

$$
\ddot{u}(\hat{t})-2 \delta \omega_{0} \dot{u}+\omega_{0}^{2}(1+\mu) u+\omega_{0}^{2} \mu u(\hat{t}+\tau)=0,
$$

in the space $\widehat{C}:=\widehat{C}\left([0, \tau], \mathbb{R}^{n}\right)$ with respect to the associated bilinear relation

$$
\left(\psi_{j}(s), \phi_{\kappa}(\theta)\right)=\left(\psi_{j}(0), \phi_{\kappa}(0)\right)-\omega_{0}^{2} \mu \int_{-\tau}^{0} \psi_{j}(\zeta+\tau) \phi_{k}(\zeta) d \zeta, \quad j, \kappa=1,2, \ldots,
$$

have eigenvalues $\lambda$ satisfying the transcendental characteristic equation

$$
\Delta(\lambda, \mu):=\lambda^{2}+2 \delta \omega_{0} \lambda+\omega_{0}^{2}\left\{1+\mu\left(1+e^{-\lambda \tau}\right)\right\}=0 .
$$

By Hopf bifurcation, we let $\lambda_{1,2}=v(\mu) \pm i \omega(\mu)$ with $v(\mu)>0, \omega(\mu) \neq 0$, and $v\left(\mu_{c}\right)=0$, $\mathbb{R} e\{d \Delta(\lambda, \mu) / d \mu\} \neq 0$, be the eigenvalues of (3.2d). All the remaining eigenvalues of $\Delta(\lambda, \mu)=0$ have negative real parts. Then, the substitution of $\lambda_{1}=v(\mu)+i \omega(\mu)$ into $\Delta(\lambda, \mu)=0$, and setting the real and imaginary parts of the resulting algebraic equations to zero, we have

$$
\begin{gathered}
\Delta(\lambda, \mu):=G(\lambda, \mu)+i M(\lambda, \mu)=0, \\
G(\lambda, \mu):=v^{2}-\omega^{2}+2 \delta \omega_{0} v+\omega_{0}^{2}\left\{1+\mu\left(1+e^{-v \tau} \cos \omega \tau\right)\right\}=0, \\
M(\lambda, \mu):=2 v \omega+2 \delta \omega_{0} \omega-\omega_{0}^{2} \mu e^{-v \tau} \sin \omega \tau=0,
\end{gathered}
$$

which by putting $v=0$ reduces to the form

$$
\begin{gathered}
G(\lambda, \mu):=\omega_{0}^{2}-\omega^{2}+\omega_{0}^{2} \mu(1+\cos \omega \tau)=0, \\
M(\lambda, \mu):=2 \delta \omega_{0} \omega-\omega_{0}^{2} \mu \sin \omega \tau=0,
\end{gathered}
$$

or equivalently

$$
\omega_{0}^{2} \mu(1+\cos \omega \tau)=-\left(\omega_{0}^{2}-\omega^{2}\right), \quad \omega_{0}^{2} \mu \sin \omega \tau=2 \delta \omega_{0} \omega
$$

Possible values of $\omega \tau$ are of the form $\omega \tau \in(2 \pi \kappa,(2 \kappa+1) \pi), \kappa=0,1,2, \ldots$, and they are such that (3.3) hold. Now, squaring both sides of (3.3c) and adding the result together will lead to

$$
2 \omega_{0}^{4} \mu^{2}(1+\cos \omega \tau)=\left(\omega_{0}^{2}-\omega^{2}\right)^{2}+\left(2 \delta \omega_{0} \omega\right)^{2},
$$


where the substitution of the first equation in (3.3c) into (3.4a) yields the explicit expression for the bifurcation parameter $\mu$ as

$$
\mu=-\frac{\left(\omega_{0}^{2}-\omega^{2}\right)^{2}+\left(2 \delta \omega_{0} \omega\right)^{2}}{2 \omega_{0}^{2}\left(\omega_{0}^{2}-\omega^{2}\right)}=-\frac{\left(1-\left(\omega / \omega_{0}\right)^{2}\right)^{2}+4 \delta^{2}\left(\omega / \omega_{0}\right)^{2}}{1-\left(\omega / \omega_{0}\right)^{2}} .
$$

We compute Hopf's transversality condition by differentiating implicitly (3.2d) with respect to $\mu$, obtaining

$$
\frac{d}{d \mu}\{\Delta(\lambda, \mu)\}=\frac{d \Delta(\lambda, \mu)}{d \lambda} \times \frac{d \lambda}{d \mu}+\frac{d \Delta(\lambda, \mu)}{d \mu}=0
$$

from which the real and imaginary parts when $\lambda=\nu+i \omega$ are given by

$$
\begin{aligned}
\left\{\frac{d \lambda}{d \mu}\right\}_{\lambda=v+i \omega} & =\left\{\left(\frac{d \Delta(\lambda, \mu)}{d \mu}\right)\left(\frac{d \Delta(\lambda, \mu)}{d \lambda}\right)^{-1}\right\}_{\lambda=v+i \omega} \\
& =\left\{-\frac{\omega_{0}^{2}\left(1+e^{-\lambda \tau}\right)}{2\left(\lambda+\delta \omega_{0}\right)-\omega_{0}^{2} \tau \mu e^{-\lambda \tau}}\right\}_{\lambda=v+i \omega} \\
& =\left\{\frac{\Delta_{111}^{(1)}(\lambda, \mu)}{\Delta_{111}^{(2)}(\lambda, \mu)}\right\}_{\lambda=v+i \omega}=\tilde{G}(\lambda, \mu)+i \widetilde{M}(\lambda, \mu), \quad i=\sqrt{-1},
\end{aligned}
$$

with the representations

$$
\begin{gathered}
\lambda^{2}+2 \delta \omega_{0} \lambda+\omega_{0}^{2}=-\omega_{0}^{2} \mu\left(1+e^{-\lambda \tau}\right), \\
\Delta_{111}^{(1)}(\lambda, \mu):=\mu^{-1}\left(\lambda^{2}+2 \delta \omega_{0} \lambda+\omega_{0}^{2}\right), \\
\widetilde{G}(\lambda, \mu):=\frac{1}{\mu\left(\beta_{111}^{2}+\beta_{112}^{2}\right)}\left\{\beta_{111}^{(2)}\left(\omega^{2}-\omega_{0}^{2}-2 \delta \omega_{0} v-v^{2}\right)+2 \omega \beta_{112}\left(v+\delta \omega_{0}\right)\right\}, \\
\widetilde{M}(\lambda, \mu):=-\frac{1}{\mu\left(\beta_{111}^{2}+\beta_{112}^{2}\right)}\left\{2 \omega \beta_{111}\left(v+\delta \omega_{0}\right)+\beta_{112}\left(\omega^{2}-\omega_{0}^{2}-2 \delta \omega_{0} v-v^{2}\right)\right\}, \\
\beta_{111}:=\tau\left(\omega^{2}-v^{2}\right)-2 \delta \omega_{0} \tau v-\omega_{0}^{2} \tau(1+\mu)+2\left(v+\delta \omega_{0}\right), \\
\beta_{112}:=-2 \omega\left(1-\tau\left(v+\delta \omega_{0}\right)\right),
\end{gathered}
$$

and putting $v=0$ into these equations will thus show that

$$
\mathbb{R e}\left(\frac{d \lambda}{d \mu}\right)_{\lambda=i \omega}=\{\tilde{G}(\lambda, \mu)\}_{\lambda=i \omega}>0
$$


if the choice of values for the model parameters are such that the inequality

$$
\mu\left(\omega^{2}-\omega_{0}^{2}\right)\left\{\tau\left(\omega_{0}^{2}(1+\mu)-\omega^{2}\right)+2 \delta \omega_{0}\right\}>4 \delta \omega_{0} \omega \mu\left(1+\delta \omega_{0} \tau\right)
$$

holds. This means that the pair of eigenvalues $\lambda_{1,2}=v(\mu) \pm i \omega(\mu)$ of $\Delta(\lambda, \mu)=0$ with $v\left(\mu_{c}\right)=0, \omega\left(\mu_{c}\right) \neq 0$ will cross the imaginary axis from left to right in the complex plane with a nonzero speed as the bifurcation parameter $\mu$ varies near its critical value $\mu_{c}$. Also, it ensures that the steady state stability of the system is lost at $\mu=\mu_{c}$, and instability in the sense of supercritical and subcritical bifurcations will persist for larger values of $\mu$. The exact nature of such bifurcations satisfying Hopf's conditions (3.3)-(3.5) will depend on the nonlinearity in the delay system.

Rewriting (3.4b) as a quadratic in $\omega^{2}$ and letting $\omega^{2}=\tilde{\omega}$, we have

$$
\widetilde{\omega}^{2}+\alpha_{1} \widetilde{\omega}^{2}+\alpha_{2}=0, \quad \alpha_{1}:=2 \omega_{0}^{2}\left(2 \delta^{2}-(1+\mu)\right), \alpha_{2}:=\omega_{0}^{4}(1+2 \mu),
$$

whose real and positive solutions are of the form

$$
\tilde{\omega}_{1}=\frac{1}{2}\left(-\alpha_{1}+\sqrt{\alpha_{1}^{2}-4 \alpha_{2}}\right), \quad \tilde{\omega}_{2}=\frac{1}{2}\left(-\alpha_{1}-\sqrt{\alpha_{1}^{2}-4 \alpha_{2}}\right),
$$

if the following hold:

$$
\begin{array}{ll}
\alpha_{1}<0 \mid 2 \delta^{2}<(1+\mu) ; & \alpha_{1}^{2}-4 \alpha_{2}>0 \mid\left\{2 \delta^{2}-(1+\mu)\right\}^{2}>(1+2 \mu), \\
\alpha_{1}<0 \mid 2 \delta^{2}<(1+\mu) ; & \alpha_{1}^{2}-4 \alpha_{2}=0 \mid\left\{2 \delta^{2}-(1+\mu)\right\}^{2}=(1+2 \mu) .
\end{array}
$$

In addition, one has to show that the substitution of $\widetilde{\omega}_{1}$ and $\widetilde{\omega}_{2}$ into (3.5) will ensure Hopf's transversality condition, that is, $\mathbb{R} e(d \lambda / d \mu)_{\lambda=i \omega}>0$. Therefore, with $\widetilde{\omega}_{1}=\omega^{2}$ and $\mathbb{R} e(d \lambda / d \mu)_{\lambda=i \omega}>0$, we obtain

$$
\begin{gathered}
2 \omega^{2}+\alpha_{1}=0 \mid \frac{\omega}{\omega_{0}}=\sqrt{-\left(2 \delta^{2}-(1+\mu)\right)}, \\
\alpha_{1}^{2}-4 \alpha_{2}=0 \mid(\mu-2 \delta(\delta+1))(\mu-2 \delta(\delta-1))=0, \\
\therefore \mu_{1}=2 \delta(1+\delta)>0, \quad 0<\delta<1, \quad \mu_{2}=2 \delta(\delta-1)>0, \quad \delta>1,
\end{gathered}
$$

where by setting $\omega_{\min }=\omega$ and $\mu_{\min }=\mu_{1}$, we have

$$
\mu_{\min }=2 \delta(1+\delta), \quad \frac{\omega_{\min }}{\omega_{0}}=\sqrt{-\left(2 \delta^{2}-\left(1+\mu_{m}\right)\right)}=\sqrt{1+2 \delta} .
$$

These are the minimum values separating the regimes of stable and unstable solutions of the linearized delay equation at the Hopf bifurcation point $\mu=\mu_{c}, \lambda_{1,2}= \pm i \omega\left(\mu_{\min }\right)=$ $\pm i \omega_{0} \sqrt{1+2 \delta}$. Below and equal to these minimum values, all the infinite number of eigenvalues of the transcendental characteristic equation (3.2d) will lie in the left-hand side of the complex plane. The minimum values are consistent with earlier computations by Campbell et al. [1], Cooke and Grossman [5], and Fofana et al. [8]. 
Next, deriving an expression for $\tau$ begins by further rewriting (3.3c) as follows:

$$
\frac{\sin \omega \tau}{1+\cos \omega \tau}=-\frac{2 \delta \omega_{0} \omega}{\omega_{0}^{2}-\omega^{2}}=-\frac{2 \delta\left(\omega / \omega_{0}\right)}{1-\left(\omega / \omega_{0}\right)^{2}}
$$

where with $\cos ^{2} \omega \tau+\sin ^{2} \omega \tau=1$ in mind and the trigonometric identity

$$
\begin{aligned}
\tan \frac{\omega \tau}{2} & =\sqrt{\frac{\sin ^{2} \omega \tau / 2}{\cos ^{2} \omega \tau / 2}}=\sqrt{\frac{1 / 2(1-\cos \omega \tau)}{1 / 2(1+\cos \omega \tau)}} \\
& =\sqrt{\frac{(1-\cos \omega \tau)(1+\cos \omega \tau)}{(1+\cos \omega \tau)(1+\cos \omega \tau)}} \\
& =\sqrt{\frac{1-\cos ^{2} \omega \tau}{(1+\cos \omega \tau)^{2}}}=\sqrt{\frac{\sin ^{2} \omega \tau}{(1+\cos \omega \tau)^{2}}} \\
& =\left|\frac{\sin \omega \tau}{1+\cos \omega \tau}\right|=\frac{\sin \omega \tau}{1+\cos \omega \tau},
\end{aligned}
$$

so that

$$
\frac{\omega \tau_{\kappa}}{2}=\arctan \left(\frac{\sin \omega \tau_{\kappa}}{1+\cos \omega \tau_{\kappa}}\right)+\pi \kappa, \quad \kappa=0,1,2,3, \ldots, n, \ldots,
$$

we obtain

$$
\tau_{\kappa}=\frac{2}{\omega}\left(\arctan \left(-\frac{2 \delta\left(\omega / \omega_{0}\right)}{1-\left(\omega / \omega_{0}\right)^{2}}\right)+\pi \kappa\right)
$$

and using the minimum value for $\omega_{\min }$ in $(3.7 \mathrm{~b})$, we get

$$
\begin{aligned}
\tau_{\kappa, \min } & =\frac{2}{\omega_{0} \sqrt{1+2 \delta}}\left(\arctan \left(-\frac{2 \delta \sqrt{1+2 \delta}}{1-(\sqrt{1+2 \delta})^{2}}\right)+\pi \kappa\right) \\
& =\frac{2}{\omega_{0} \sqrt{1+2 \delta}}(\arctan (\sqrt{1+2 \delta})+\pi \kappa) \quad \kappa=0,1,2,3, \ldots, n, \ldots
\end{aligned}
$$

With these minimum values for the gain $\mu_{\min }$, time delay $\tau_{\kappa, \min }$, and the response frequency $\omega_{\min }=\omega_{0} \sqrt{1+2 \delta}$, one can describe all the critical settings of the model parameters in the delay equation (3.1) at which unstable-free oscillation persists. Should unstable oscillations appear at some desired frequency such that $\omega_{\min }=\omega_{0} \sqrt{1+2 \delta}>1$, fine-tuning the model parameters in the vicinity of their critical settings can control such oscillations. To find out whether the bifurcations are supercritical and/or subcritical, we carry out a local centre manifold analysis of the nonlinear delay equation (3.1) as presented in the preceding section. 
3.2. Nonlinear analysis. We begin by defining the elements of the initial continuous function $\phi(\theta) \in C$ and its projections $\phi^{P}(\theta), \phi^{Q}(\theta)$ onto the centre and stable subspaces $P, Q \in C$. For the eigenvalues of $\lambda= \pm i \omega$, we have $\phi^{P}(\theta)=\Phi(\theta) b$ with $\phi_{\kappa}^{P}(\theta)=e^{\lambda_{\kappa} \theta},-\tau \leq$ $\theta \leq 0$ of the generalized eigenspace $P \in C$ and $\psi^{\hat{P}}(s)=\Psi(s) \hat{b}, \psi_{j}^{\hat{P}}(s) e^{-\lambda_{j} s}, 0 \leq s \leq \tau, j=$ $\kappa=1,2$ of the adjoint generalized eigenspace $\hat{P} \in \hat{C}$. The values of the basis $\Phi(\theta) \in C$ for $P$ is thus given by

$$
\begin{aligned}
\Phi(\theta) b & =\left[\phi_{1}(\theta), \phi_{2}(\theta)\right]=c_{1} e^{i \omega \theta}\left[\begin{array}{c}
1 \\
-i \omega
\end{array}\right]+c_{2} e^{-i \omega \theta}\left[\begin{array}{c}
1 \\
i \omega
\end{array}\right] \\
& =\left[\begin{array}{c}
c_{1} e^{i \omega}+c_{2} e^{-i \omega} \\
-i \omega c_{1} e^{i \omega}+i \omega c_{2} e^{-i \omega}
\end{array}\right], \quad-\tau \leq \theta \leq 0, \\
& =\left[\begin{array}{cc}
\left(c_{1}+c_{2}\right) \cos \omega \theta & i\left(c_{1}-c_{2}\right) \sin \omega \theta \\
\omega\left(c_{1}+c_{2}\right) \sin \omega \theta & -i \omega\left(c_{1}-c_{2}\right) \cos \omega \theta
\end{array}\right]\left[\begin{array}{cc}
\cos \omega \theta & -\sin \omega \theta \\
\omega \sin \omega \theta & \omega \cos \omega \theta
\end{array}\right]\left[\begin{array}{l}
b_{1} \\
b_{2}
\end{array}\right],
\end{aligned}
$$

where

$$
c_{1}:=p+i q, \quad c_{2}:=p-i q \mid c_{1}+c_{2}=2 p:=b_{1}, \quad i\left(c_{1}-c_{2}\right)=-2 q:=-b_{2},
$$

and similarly the basis $\Psi(s) \in \hat{C}$ for $\widehat{P}$ is of the form

$$
\Psi(s)=\left[\psi_{1}(s), \psi_{2}(s)\right]^{T}=\left[\begin{array}{cc}
\cos \omega s & \omega \sin \omega s \\
-\sin \omega s & \omega \cos \omega s
\end{array}\right], \quad 0 \leq s \leq \tau .
$$

These bases form the inner product matrix

$$
\begin{aligned}
(\Psi(s), \Phi(\theta)) & =\left[\begin{array}{l}
\psi_{1}(s) \\
\psi_{2}(s)
\end{array}\right]\left[\begin{array}{ll}
\phi_{1}(\theta) & \phi_{2}(\theta)
\end{array}\right] \\
& =\left[\begin{array}{ll}
\left(\psi_{1}(s) \phi_{1}(\theta)\right) & \left(\psi_{1}(s) \phi_{2}(\theta)\right) \\
\left(\psi_{2}(s) \phi_{1}(\theta)\right) & \left(\psi_{2}(s) \phi_{2}(\theta)\right)
\end{array}\right],
\end{aligned}
$$

in which

$$
\begin{aligned}
& \left(\psi_{1}(s) \phi_{1}(\theta)\right)=\left(\cos \omega s \cos \omega \theta+\omega^{2} \sin \omega s \sin \omega \theta\right), \\
& \left(\psi_{1}(s) \phi_{2}(\theta)\right)=-\left(\cos \omega s \sin \omega \theta-\omega^{2} \sin \omega s \cos \omega \theta\right), \\
& \left(\psi_{2}(s) \phi_{1}(\theta)\right)=-\left(\sin \omega s \cos \omega \theta-\omega^{2} \cos \omega s \sin \omega \theta\right), \\
& \left(\psi_{2}(s) \phi_{2}(\theta)\right)=\left(\sin \omega s \sin \omega \theta+\omega^{2} \cos \omega s \sin \omega \theta\right) .
\end{aligned}
$$


The substitution of the elements of $(\Psi(s), \Phi(\theta))$ into the bilinear relation (3.2c) yields the nonsingular matrix (i.e., $\left.\operatorname{det}(\Psi, \Phi)_{\text {nsg }} \neq 0\right)$

$$
(\Psi, \Phi)_{\mathrm{nsg}}=\left[\begin{array}{ll}
\left(\psi_{1}, \phi_{1}\right) & \left(\psi_{1}, \phi_{2}\right) \\
\left(\psi_{2}, \phi_{1}\right) & \left(\psi_{2}, \phi_{2}\right)
\end{array}\right]
$$

where

$$
\begin{gathered}
\left(\psi_{1}, \phi_{1}\right)=1-\frac{1}{2} \omega_{0}^{2} \mu\left\{\left(\frac{1}{\omega}-\omega\right) \sin \omega \tau+\tau\left(1+\omega^{2}\right) \cos \omega \tau\right\} \\
\left(\psi_{1}, \phi_{2}\right)=-\frac{1}{2} \omega_{0}^{2} \tau \mu\left(1+\omega^{2}\right) \sin \omega \tau, \\
\left(\psi_{2}, \phi_{1}\right)=\frac{1}{2} \omega_{0}^{2} \tau \mu\left(1+\omega^{2}\right) \sin \omega \tau, \\
\left(\psi_{2}, \phi_{2}\right)=\omega^{2}+\frac{1}{2} \omega_{0}^{2} \mu\left\{\left(\frac{1}{\omega}-\omega\right) \sin \omega \tau-\tau\left(1+\omega^{2}\right) \cos \omega \tau\right\} .
\end{gathered}
$$

Then, the basis $\Psi(s)$ for $\hat{P} \in \hat{C}$ of the adjoint equation (3.2b) is normalized to $\bar{\Psi}(s)=$ $\left[\bar{\psi}_{1}(s), \bar{\psi}_{2}(s)\right]^{T} \in \hat{C}$, computing $\bar{\Psi}(s)=(\Psi, \Phi)_{\text {nsg }}^{-1} \Psi(s)$ to yield

$$
\begin{gathered}
\bar{\Psi}(s)=\left[\begin{array}{ll}
\bar{\psi}_{11}(s) & \bar{\psi}_{12}(s) \\
\bar{\psi}_{21}(s) & \bar{\psi}_{22}(s)
\end{array}\right], \quad 0 \leq s \leq \tau, \\
\bar{\psi}_{11}(s)=\frac{1}{\operatorname{det}\left((\Psi, \Phi)_{\mathrm{nsg}}^{-1}\right)}\left\{\left(\psi_{2}, \phi_{2}\right) \cos \omega s+\left(\psi_{1}, \phi_{2}\right) \sin \omega s\right\}, \\
\bar{\psi}_{12}(s)=\frac{\omega}{\operatorname{det}\left((\Psi, \Phi)_{\mathrm{nsg}}^{-1}\right)}\left\{\left(\psi_{2}, \phi_{2}\right) \sin \omega s-\left(\psi_{1}, \phi_{2}\right) \cos \omega s\right\}, \\
\bar{\psi}_{21}(s)=-\frac{1}{\operatorname{det}\left((\Psi, \Phi)_{\mathrm{nsg}}^{-1}\right)}\left\{\left(\psi_{2}, \phi_{1}\right) \cos \omega s+\left(\psi_{1}, \phi_{1}\right) \sin \omega s\right\}, \\
\bar{\psi}_{22}(s)=-\frac{\omega}{\operatorname{det}\left((\Psi, \Phi)_{\mathrm{nsg}}^{-1}\right)}\left\{\left(\psi_{2}, \phi_{1}\right) \sin \omega s-\left(\psi_{1}, \phi_{1}\right) \cos \omega s\right\}, \\
\operatorname{det}\left((\Psi, \Phi)_{\mathrm{nsg}}^{-1}\right)=\left\{\left(\psi_{1}, \phi_{1}\right)\left(\psi_{2}, \phi_{2}\right)-\left(\psi_{1}, \phi_{2}\right)\left(\psi_{2}, \phi_{1}\right)\right\},
\end{gathered}
$$

where the substitution of the new elements $\left(\psi_{j}(s), \phi_{k}(\theta)\right), j, k=1,2$ into (3.2c) will lead to the identity matrix

$$
(\Psi, \Phi)_{\mathrm{id}}=\frac{1}{\operatorname{det}\left((\Psi, \Phi)_{\mathrm{nsg}}^{-1}\right)}\left[\begin{array}{cc}
\operatorname{det}\left((\Psi, \Phi)_{\mathrm{nsg}}^{-1}\right) & 0 \\
0 & \operatorname{det}\left((\Psi, \Phi)_{\mathrm{nsg}}^{-1}\right)
\end{array}\right]=\left[\begin{array}{ll}
1 & 0 \\
0 & 1
\end{array}\right]
$$

Consequently, the constant matrices $B \in C$ and $\widehat{B} \in \hat{C}$ are equivalent, and the elements of $B$ at Hopf bifurcation are $B=\left[[0, \omega]^{T},[-\omega, 0]^{T}\right]$. 
With the algebraic simplifications

$$
\begin{aligned}
e^{B \theta}= & I+B \theta+\frac{(B \theta)^{2}}{2}+\frac{(B \theta)^{3}}{3 !}+\frac{(B \theta)^{4}}{4 !}+\frac{(B \theta)^{5}}{5 !}+\cdots \\
= & {\left[\begin{array}{ll}
1 & 0 \\
0 & 1
\end{array}\right]+\theta\left[\begin{array}{cc}
0 & -\omega \\
\omega & 0
\end{array}\right]+\frac{\theta^{2}}{2}\left[\begin{array}{cc}
0 & -\omega \\
\omega & 0
\end{array}\right]^{2} } \\
& +\frac{\theta^{3}}{6}\left[\begin{array}{cc}
0 & -\omega \\
\omega & 0
\end{array}\right]^{3}+\frac{\theta^{4}}{24}\left[\begin{array}{cc}
0 & -\omega \\
\omega & 0
\end{array}\right]^{4}+\frac{\theta^{5}}{120}\left[\begin{array}{cc}
0 & -\omega \\
\omega & 0
\end{array}\right]^{5}+\cdots \\
= & {\left[\begin{array}{cc}
\left(1-\frac{\omega^{2} \theta^{2}}{2}+\cdots\right) & -\left(\omega \theta-\frac{\omega^{3} \theta^{3}}{6}+\cdots\right) \\
\left(\omega \theta-\frac{\omega^{3} \theta^{3}}{6}+\cdots\right) & \left(1-\frac{\omega^{2} \theta^{2}}{2}+\cdots\right)
\end{array}\right]=\left[\begin{array}{cc}
\cos \omega \theta & -\sin \omega \theta \\
\sin \omega \theta & \cos \omega \theta
\end{array}\right], }
\end{aligned}
$$

one can easily see that

$$
\begin{aligned}
\Phi(\theta)=\Phi(0) e^{B \theta} & =\left[\begin{array}{cc}
\cos (0) & -\sin (0) \\
\omega \sin (0) & \omega \cos (0)
\end{array}\right]\left[\begin{array}{cc}
\cos \omega \theta & -\sin \omega \theta \\
\sin \omega \theta & \cos \omega \theta
\end{array}\right] \\
& =\left[\begin{array}{ll}
1 & 0 \\
0 & \omega
\end{array}\right]\left[\begin{array}{cc}
\cos \omega \theta & -\sin \omega \theta \\
\sin \omega \theta & \cos \omega \theta
\end{array}\right] \\
& =\left[\begin{array}{cc}
\cos \omega \theta & -\sin \omega \theta \\
\omega \sin \omega \theta & \omega \cos \omega \theta
\end{array}\right], \quad-\tau \leq \theta \leq 0,
\end{aligned}
$$

and it follows that $J(t, \mu) \Phi(\theta)=\Phi(0) e^{B(\theta+t)}$ is indeed the solution operator of the linearized delay equations in $C$. Similarly, we have $\bar{\Psi}(s)=\bar{\Psi}(0) e^{-\widehat{B} s}, 0 \leq s \leq \tau$ and $\hat{J}(\hat{t}, \mu) \bar{\Psi}(s)$ $=\bar{\Psi}(0) e^{\hat{B}(\hat{t}+s)}$ is the solution operator for the adjoint delay equations in $\widehat{C}$.

By the change of variables $x_{t}^{P}(\theta, \mu, \varepsilon)=\Phi(\theta) y(t)+x_{t}^{Q}(\theta, \mu, \varepsilon)$ with $y(t) \in \mathbb{R}^{2}, y(t)=$ $\left(\bar{\Psi}(s), \phi^{P}(\theta)\right)$, we obtain as the following a first-order approximation in $\varepsilon$, for $\theta=-\tau$,

$$
\begin{aligned}
{\left[\begin{array}{l}
x_{1}(t-\tau) \\
x_{2}(t-\tau)
\end{array}\right] } & =\left[\begin{array}{cc}
\cos \omega(-\tau) & -\sin \omega(-\tau) \\
\omega \sin \omega(-\tau) & \omega \cos \omega(-\tau)
\end{array}\right]\left[\begin{array}{l}
y_{1}(t) \\
y_{2}(t)
\end{array}\right] \\
& =\left[\begin{array}{cc}
\cos \omega \tau & \sin \omega \tau \\
-\omega \sin \omega \tau & \omega \cos \omega \tau
\end{array}\right]\left[\begin{array}{l}
y_{1}(t) \\
y_{2}(t)
\end{array}\right] \\
& =\left[\begin{array}{c}
y_{1}(t) \cos \omega \tau+y_{2}(t) \sin \omega \tau \\
-y_{1}(t) \omega \sin \omega \tau+y_{2}(t) \omega \cos \omega \tau
\end{array}\right],
\end{aligned}
$$

and for $\theta=0$,

$$
\begin{aligned}
{\left[\begin{array}{l}
x_{1}(t-0) \\
x_{2}(t-0)
\end{array}\right] } & =\left[\begin{array}{cc}
\cos \omega(0) & -\sin \omega(0) \\
\omega \sin \omega(0) & \omega \cos \omega(0)
\end{array}\right]\left[\begin{array}{l}
y_{1}(t) \\
y_{2}(t)
\end{array}\right] \\
& =\left[\begin{array}{ll}
1 & 0 \\
0 & \omega
\end{array}\right]\left[\begin{array}{l}
y_{1}(t) \\
y_{2}(t)
\end{array}\right]=\left[\begin{array}{c}
y_{1}(t) \\
\omega y_{2}(t)
\end{array}\right] .
\end{aligned}
$$


Hence, the centre manifold ODEs in $C\left([-\tau, 0], \mathbb{R}^{n}\right)$ are of the form

$$
\begin{gathered}
\dot{y}_{1}(t)=-\omega y_{2}-\varepsilon \omega_{0}^{2} \bar{\psi}_{12}(0)\left\{\sigma_{3}\left(y_{1}(1+\cos \omega \tau)+y_{2} \sin \omega \tau\right)^{3}+\tilde{\mu}\left(y_{1}(1+\cos \omega \tau)+y_{2} \sin \omega \tau\right)\right\}, \\
\dot{y}_{2}(t)=\omega y_{1}-\varepsilon \omega_{0}^{2} \bar{\psi}_{22}(0)\left\{\sigma_{3}\left(y_{1}(1+\cos \omega \tau)+y_{2} \sin \omega \tau\right)^{3}+\tilde{\mu}\left(y_{1}(1+\cos \omega \tau)+y_{2} \sin \omega \tau\right)\right\},
\end{gathered}
$$

where $\bar{\psi}_{12}(0), \bar{\psi}_{22}(0)$ are the values for $s=0$ in the normalized function $\bar{\Psi}(s) \in \widehat{C}([0, \tau]$, $\left.\mathbb{R}^{n}\right)$ for all values of $t \in[-\tau, \infty)$. By using the transformation

$$
z_{1}=y_{1}-m \omega y_{2}, \quad z_{2}=m \omega y_{1}+y_{2}, \quad m:=\frac{\bar{\psi}_{12}(0)}{\omega \bar{\psi}_{22}(0)},
$$

so that the substitution of

$$
y_{1}=\frac{1}{1+m^{2} \omega^{2}}\left(z_{1}+m \omega z_{2}\right), \quad y_{2}=-\frac{1}{1+m^{2} \omega^{2}}\left(m \omega z_{1}-z_{2}\right),
$$

into the perturbation

$$
g\left(y_{1}, y_{2}, \mu, \varepsilon\right):=\sigma_{3} \omega_{0}^{2}\left(y_{1}(1+\cos \omega \tau)+y_{2} \sin \omega \tau\right)^{3}+\tilde{\mu} \omega_{0}^{2}\left(y_{1}(1+\cos \omega \tau)+y_{2} \sin \omega \tau\right),
$$

we obtain

$$
\begin{aligned}
G\left(z_{1}, z_{2}, \mu, \varepsilon\right):= & \frac{\sigma_{3} \omega_{0}^{2}}{\left(1+m^{2} \omega^{2}\right)^{3}}\left\{\left(z_{1}+m \omega z_{2}\right)(1+\cos \omega \tau)-\left(m \omega z_{1}-z_{2}\right) \sin \omega \tau\right\}^{3} \\
& +\tilde{\mu} \omega_{0}^{2}\left\{\left(z_{1}+m \omega z_{2}\right)(1+\cos \omega \tau)-\left(m \omega z_{1}-z_{2}\right) \sin \omega \tau\right\} .
\end{aligned}
$$

Hence, the centre manifold ODEs are further written into the form

$$
\begin{aligned}
& \dot{z}_{1}(t)=-\omega z_{2}, \\
& \dot{z}_{2}(t)=\omega z_{1}-\varepsilon \frac{\bar{\psi}_{12}^{2}(0)+\bar{\psi}_{22}^{2}(0)}{\bar{\psi}_{22}(0)} G\left(z_{1}, z_{2}, \mu, \varepsilon\right) .
\end{aligned}
$$

Bifurcation scenarios of (3.17) leading to stable and unstable regimes can be described using standard mathematical tools of nonlinear dynamics [9].

\section{The van der Pol equation with delay}

Consider the infinite-dimensional reduction of the delay version of the van der Pol-type equation

$$
\ddot{x}(t)+\varepsilon \beta_{3}\left(x^{2}(t-\tau)-2 \delta \omega_{0}\right) \dot{x}(t-\tau)+\omega_{0}^{2} x(t)=0
$$

in $C:=C\left([-\tau, 0], \mathbb{R}^{n}\right)$, where $\beta_{3}$ is a real coefficient representing the perturbation. The linearized part of (4.1) in $C$ and its corresponding adjoint form in $\hat{C}\left([0, \tau], \mathbb{R}^{n}\right)$ have eigenvalues $\lambda$ satisfying the characteristic equation $\Delta:=\lambda^{2}+\omega_{0}^{2}=0$. Clearly, $\lambda_{1,2}= \pm i \omega_{0}$ 
are eigenvalues of $\Delta=0$, and the corresponding bases $\Phi(\theta)$ of $P \in C$ and $\Psi(s)$ of $\hat{P} \in \hat{C}$ are therefore given by

$$
\begin{aligned}
& \Phi(\theta)=\left[\phi_{1}(\theta), \phi_{2}(\theta)\right]=\left[\begin{array}{cc}
\cos \omega \theta & \left(\omega_{0}\right)^{-1} \sin \omega \theta \\
-\omega \operatorname{sub} 0 \sin \omega \theta & \cos \omega \theta
\end{array}\right], \quad-\tau \leq \theta \leq 0, \\
& \Psi(s)=\left[\psi_{1}(s), \psi_{2}(s)\right]^{T}=\left[\begin{array}{cc}
\cos \omega s & -\omega \operatorname{sub} 0 \sin \omega s \\
\left(\omega_{0}\right)^{-1} \sin \omega \theta & \cos \omega s
\end{array}\right], \quad 0 \leq s \leq \tau .
\end{aligned}
$$

Proceeding exactly as above, the change of variables $x_{t}^{P}(\theta)=\Phi(\theta) y(t)+x_{t}^{Q}(\phi(\theta), \mu, \varepsilon)$, $y(t) \in \mathbb{R}^{2}$ will give rise to

$$
\begin{gathered}
x_{1}(t)=y_{1}(t), \quad x_{2}(t)=y_{2}(t), \quad \theta=0, \\
x_{1}(t-\tau)=y_{1} \cos \omega_{0} \tau-y_{2}\left(\omega_{0}\right)^{-1} \sin \omega_{0} \tau, \\
x_{2}(t-\tau)=y_{1} \omega_{0} \sin \omega_{0} \tau+y_{2} \cos \omega_{0} \tau, \quad \theta=-\tau .
\end{gathered}
$$

The equivalent centre manifold ODEs for the delay version van der Pol equation (3.16) are thus given by

$$
\begin{aligned}
\dot{y}_{1}(t)= & y_{2}, \\
\dot{y}_{2}(t)= & -\omega_{0}^{2} y_{1}-\varepsilon \beta_{3}\left\{\left(y_{1} \cos \omega_{0} \tau-\frac{1}{\omega_{0}} y_{2} \sin \omega_{0} \tau\right)^{2}-2 \delta \omega_{0}\right\} \\
& \times\left\{y_{1} \omega_{0} \sin \omega_{0} \tau+y_{2} \cos \omega_{0} \tau\right\} .
\end{aligned}
$$

Again, possible bifurcations can be determined for different values of the time delay $\tau>0$.

\section{Conclusion}

The role of the centre manifold theorem and Hopf bifurcation theorem in the infinitedimensional reduction of DDEs to finite-dimensional ODEs has been established. The underlying mathematical arguments of the reduction approach based upon the work by Hale [10] are presented. The time delay versions of the Duffing and van der Pol equations are considered as illustrative examples.

\section{References}

[1] S. A. Campbell, J. Bélair, T. Ohira, and J. Milton, Limit cycles, tori, and complex dynamics in a second-order differential equation with delayed negative feedback, J. Dynam. Differential Equations 7 (1995), no. 1, 213-236.

[2] S. T. Chiriacescu, Stability in the Dynamics of Metal Cutting, Elsevier, Amsterdam, 1990.

[3] S. N. Chow and J. Mallet-Paret, Integral averaging and bifurcation, J. Differential Equations 26 (1977), no. 1, 112-159.

[4] E. N. Chukwu, Stability and Time-Optimal Control of Hereditary Systems, Mathematics in Science and Engineering, vol. 188, Academic Press, Massachusetts, 1992.

[5] K. L. Cooke and Z. Grossman, Discrete delay, distributed delay and stability switches, J. Math. Anal. Appl. 86 (1982), no. 2, 592-627.

[6] L. E. Èl'sgol'ts and S. B. Norkin, Introduction to the Theory and Application of Differential Equations with Deviating Arguments, Academic Press, New York, 1973. 
[7] M. S. Fofana, Aspects of stable and unstable machining by Hopf bifurcation, Journal of Applied Mathematical Modelling 26 (2002), 953-973.

[8] M. S. Fofana, B. Q. Cabell, N. Vawter, M. F. Usilton, and P. B. Ryba, Illustrative applications of the theory of delay dynamical systems, Math. Comput. Modelling 37 (2003), no. 12-13, $1371-1382$.

[9] J. Guckenheimer and P. Holmes, Nonlinear Oscillations, Dynamical Systems, and Bifurcations of Vector Fields, Applied Mathematical Sciences, vol. 42, Springer-Verlag, New York, 1983.

[10] J. K. Hale, Theory of Functional Differential Equations, Springer-Verlag, New York, 1977.

[11] J. K. Hale and S. M. Verduyn Lunel, Introduction to Functional-Differential Equations, Applied Mathematical Sciences, vol. 99, Springer-Verlag, New York, 1993.

[12] B. D. Hassard, N. D. Kazarinoff, and Y.-H. Wan, Theory and Applications of Hopf Bifurcation, London Mathematical Society Lecture Note Series, vol. 41, Cambridge University Press, Cambridge, 1981.

[13] J. Kurzweil, Small delays don't matter, Lecture Notes in Mathematics, Springer-Verlag, New York, 1971, pp. 47-49.

[14] A. Mazanov and K. P. Tognetti, Taylor series expansion of delay differential equations-a warning, J. Theor. Biol. 46 (1974), no. 1, 271-282.

[15] F. C. Moon (ed.), Dynamics and Chaos in Manufacturing Processes, John Wiley \& Sons, New York, 1998.

[16] T. T. Soong, Active Structural Control: Theory and Practice, Longman Scientific \& Technical, Harlow; John Wiley \& Sons, New York, 1990.

[17] S. A. Tobias, Machine-Tool Vibration, Blackie and Sons, London, 1965.

M. S. Fofana: Integrative Manufacturing Control and Dynamics, Mechanical Engineering Department, Worcester Polytechnic Institute, Worcester, MA 01609-2280, USA

E-mail address: msfofana@wpi.edu 


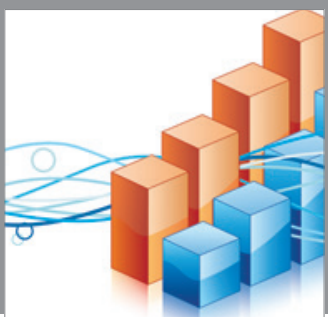

Advances in

Operations Research

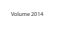

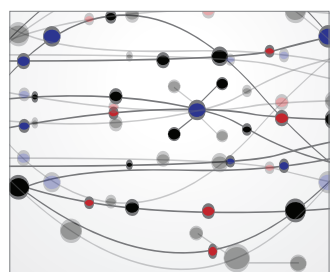

\section{The Scientific} World Journal
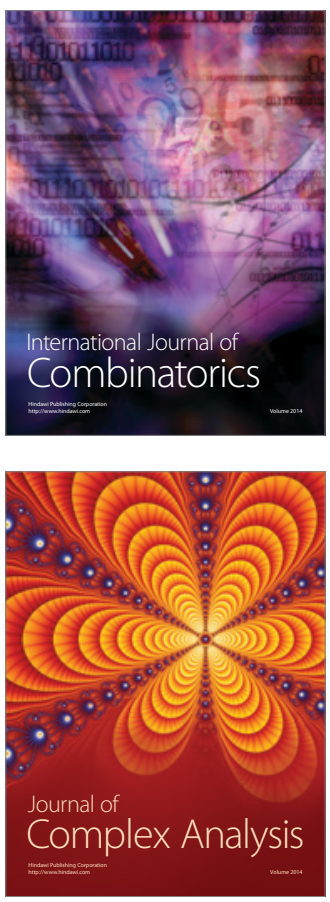

International Journal of

Mathematics and

Mathematical

Sciences
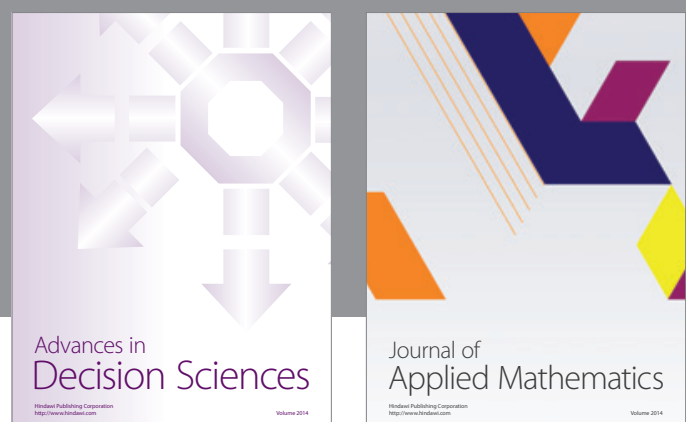

Journal of

Applied Mathematics
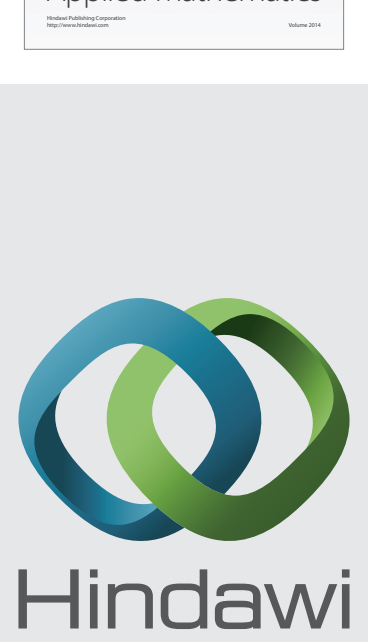

Submit your manuscripts at http://www.hindawi.com
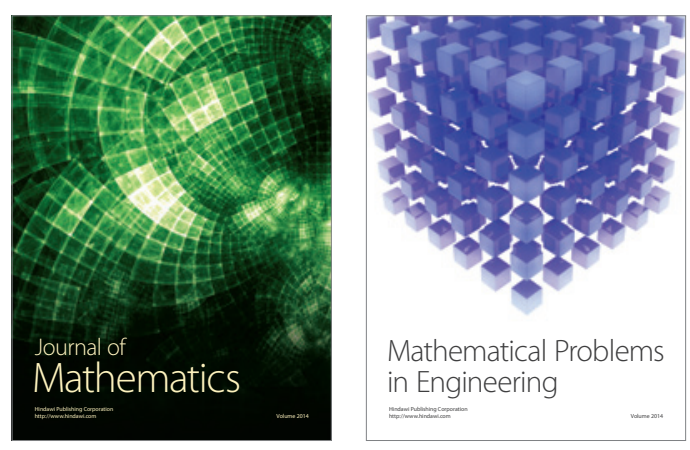

Mathematical Problems in Engineering
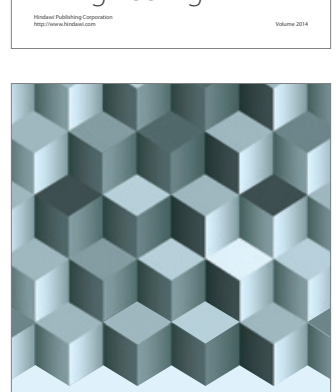

Journal of

Function Spaces
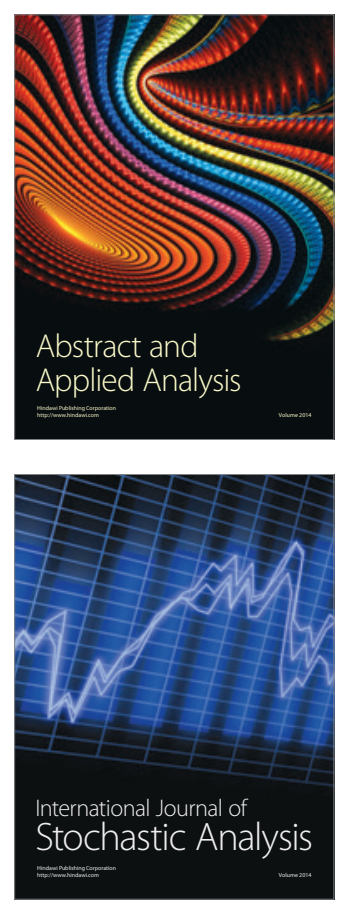

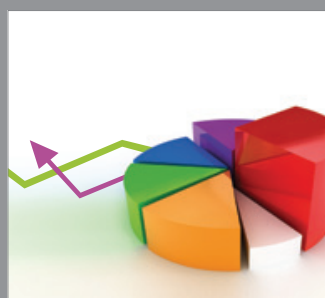

ournal of

Probability and Statistics

Promensencen
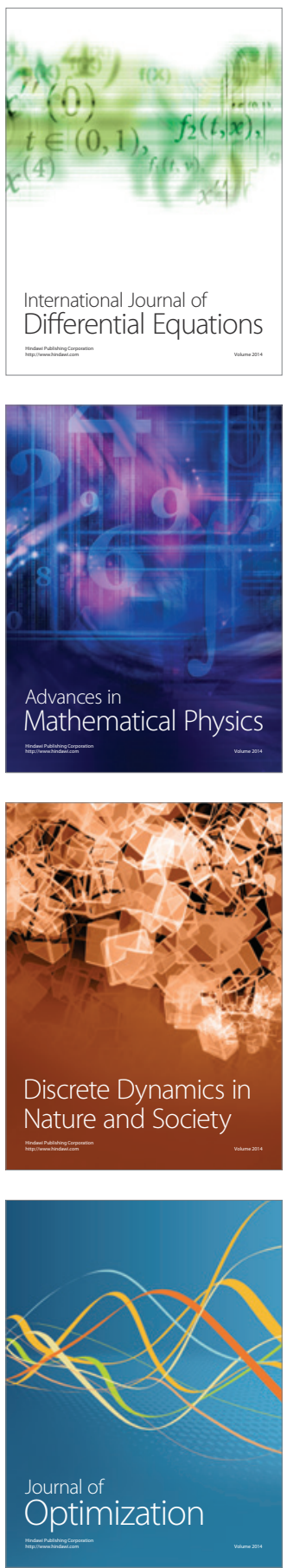